\title{
DAMPING RING FOR GENERATION OF POSITRONIUMS AND INVESTIGATION OF FERMI DEGENERATION IN MOVING BEAMS
}

\author{
A. Mikhailichenko, Cornell University, LEPP, Ithaca NY 14853, USA
}

Abstract

Kayak-paddle look-like damping ring developed as injector for a laser driven linear accelerator with microstructures [1], allows beam emittance, which is defined by quantum effects in electron-positron gas of particles in the bunch. Utilization of co-directionally moving congruent electron and positron bunches allowing production of positronium beam with $\sim 10^{10}$ parapositroniums per second. This arrangement might be a step toward investigation of the nature of Fermidegeneration in moving beams.

\section{INTRODUCTION}

The question about lowest possible emittance for moving bunch was initiated in [1], [2]. This subject has crucial importance for acceleration in microstructures, scaled down to the laser wavelengths. Here also might be a chance for enhancement for all linear collider schemes, however. One can see, that for every scheme of LC remaining under development now, utilization of beams with smaller transverse and longitudinal emittances allows to have smaller beam size at IP. So even with lowered bunch population, luminosity can be kept at the same level if emittance reduced. Lowering the number of particles in the bunch makes the beam more stable during acceleration. Lowering the bunch population also reduces a problem with intra-beam scattering phenomena in damping ring-injector.

Extreme among coolers was so called Kayak-paddle cooler first described in [3] and accommodated for laser driven linac business in [4]. It was found, that, formally, minimal phase volume occupied by single particle is limited by opening angle of radiation only and might be very close to the $\lambda_{c}=\hbar / m c$. Namely this fact forced us at this point to investigate quantum limitations associated with Fermi nature of electron gas.

\section{MINIMAL NUMBER OF PARTICLES}

Minimal number of particles required for collision can be defined as the following. In a moving frame the minimal uncertainty in definition of transverse position can be estimated as a $\lambda_{c}=\hbar / m c$-Compton wavelength, leaving uncertainty for transverse momentum as $m c$. Meanwhile spread of transverse momentum for colliding beam at IP is $p_{\perp} \cong m c \gamma \sqrt{\boldsymbol{\gamma} / \boldsymbol{\gamma} \boldsymbol{\beta}_{0}}$. For typical emittance value $\gamma \mathcal{E} \cong 10^{-4} \mathrm{~cm} \cdot \mathrm{rad}, \quad \gamma \cong 10^{6}, \quad \beta_{0} \cong 10^{-2} \mathrm{~cm}$-beta function at IP, $p_{\perp} \cong m c \cdot 10^{2}$, i.e. much more than defined by uncertainty. Minimum number of particles $N$ can be found from luminosity required $N^{2} \geq 4 \pi \lambda_{c}^{2} L / n f$, where $f$ is a repetition rate, $n$ is a number of bunches per train.
For $L \cong 10^{34}, f=100 \mathrm{~Hz}, n=10, N \geq 4 \cdot 10^{5}$. So only half of a million particles is enough for successful operation. With such amount of particles all collective phenomena vanished. We also mentioned in [4] that the lowered emittance with reduced number of particles can be obtained by scrapping all extra particles obtained from usual beam injectors. Phase density will remain the same however.

RF structure-to beam energy transfer reduction is not a problem as the productivity of equipment estimated by resulting luminosity. Lowering the bunch population increases effective accelerating gradient due to lower RF loading and less phase shift required by BNS mechanism.

\section{FERMI-DEGENERATION}

Naturally, electron gas when it is cooled will be crystallized if it is not neutralized. There are two ways for neutralization. First is neutralization by ions, second one usage of co-directionally moving electron-positron bunches. In last case creation of positroniums is the main process accompanying the cooling.

For neutralization by ions for bunch with electron density $n_{b} \cong N / \pi \sigma_{\perp}^{2} l_{b}, l_{b}$ is the bunch length, $\sigma_{\perp}$ is transverse dimension, ion density $n_{i}$, must be $Z_{i} n_{i} \gamma=n_{b} / \gamma, e Z_{i}$ - ions charge. It yields that ion density small value as $n_{i}=n_{b} / \gamma^{2} / Z_{i} \cong 10^{-7} n_{b}$ for $\gamma \cong 10^{3}, Z_{i} \cong 10$. This defined by emittance dilution as a result of collisions during damping time.

Ideal electron gas defined as a having no interactions between particles [6]. As the collisions do not change distributions as far as they are elastic, they just equalizing the temperature among degrees of freedom. That is why Fermi-degeneration of electron gas can be described so as the electrons have no interactions.

It was shown in [1] for the first time, that there exists a strong quantum limitation for the lowest emittances in the beam like

$\left(\gamma \mathcal{E}_{x}\right)\left(\gamma \mathcal{E}_{y}\right)\left(\gamma \mathcal{E}_{s}\right)=\left(\gamma \mathcal{E}_{x}\right)\left(\gamma \mathcal{E}_{y}\right)\left(\gamma l_{b}\left(\Delta p / p_{0}\right)\right) \geq \frac{1}{2}\left(2 \pi \lambda_{C}\right)^{3} N$, (1) where $\left(\gamma \varepsilon_{s}\right)=\mathcal{H}_{b}\left(\Delta p / p_{0}\right)$-is an invariant longitudinal emittance, $\Delta p / p_{0}$-is a relative momentum spread in the bunch, $\gamma \mathcal{E}_{x}$ and $\gamma \mathcal{E}_{y}$-are the transverse horizontal and vertical emittances. Formula (1) describes the electron gas trapped in limited space volume and each particle can reach every point in the phase space. Electron gas temperature $T$ in case of disperse motion can be written similar to as it was done in [1] 


$$
\frac{3}{2} N k_{B} T \cong N \cdot m c^{2} \gamma\left[\frac{\gamma \varepsilon_{x}}{\beta_{x}}+\frac{\gamma \mathcal{E}_{y}}{\beta_{y}}+\gamma\left(\frac{1}{\gamma^{2}}-\eta\left(\frac{\Delta p_{\|}}{p_{0}}\right)^{2}\right] .\right.
$$

Basically the difference connected with the fact that longitudinal mass is $m_{\|}^{-1}=\frac{1}{m \gamma}\left(\frac{1}{\gamma^{2}}-\eta\right), \eta=\frac{\gamma}{l} \frac{\partial l}{\partial \gamma}$. One can easily recognized here invariants introduced in [7]. So, these invariants are just temperature of moving bunch.

What is important here is that $3 \mathrm{D}$ case can be convolved in 2D one by operation at critical energy only, when $\eta=1 / \gamma^{2}$. In this case longitudinal temperature formally becomes equal to zero. As the auto-phasing is not working here, stability can be arranged by feedbacks. Linear motion like in pure focusing channel formally also cannot be treated like 2D, due to $\eta \neq 0$ in this case. For a two-dimensional phase space emittances now limited by

$$
\left(\gamma \varepsilon_{x}\right)\left(\gamma \varepsilon_{y}\right) \geq(1 / 2)\left(2 \pi \lambda_{C}\right)^{2} N
$$

The last also could be obtained from (1) supposing $\Delta p \cong \hbar / l_{b}$. For equal emittances this comes to $\gamma \mathcal{E}_{x} \geq 2 \pi \lambda_{C} \sqrt{N / 2}=1.7 \cdot 10^{1} \sqrt{N}$. For $N=10^{10}, \gamma \mathcal{E}_{x} \geq 1.7 \cdot 10^{-6}$ $\mathrm{cm}$ rad. We will see lower, that Kayak-Paddle cooler has adequate emittances. Fermi energy is [6]

$$
E_{F}=\frac{p_{F}^{2}}{2 m} \cong\left(3 \pi^{2}\right)^{2 / 3} \frac{\hbar^{2}}{2 m} \rho^{2 / 3} \text { or } E_{F}=c p_{F}=\left(3 \pi^{2}\right)^{1 / 3} \hbar c \rho^{1 / 3} \text {, }
$$

where $\rho=N / \sigma_{\perp}^{2} / l_{b}$ is the bunch density. The last works if the particles in the rest frame are relativistic. So for degeneration as the temperature must be $k_{B} T \leq E_{F}$. Fermi energy must be small compared with Coulomb's energy of interaction between particles, so $e^{2} / \rho^{1 / 3}<<E_{F}$. Substitute here (4) one can obtain that $\rho>>\left(\alpha / \lambda_{C}\right)^{3}$, $\alpha=e^{2} / \hbar c \cong 1 / 137$. The last condition well satisfied at high densities.

\section{KAYAK-PADDLE COOLER}

This type of cooler was introduced in [3] and described in [4]. Here we will give more details on its hardware.

Specific shape of this cooler naturally gave its name.

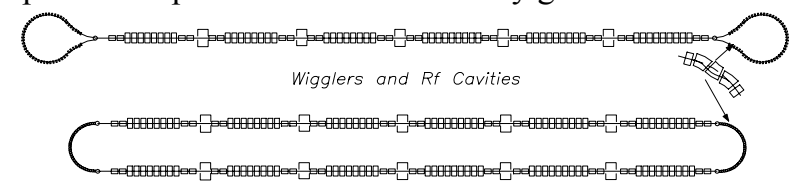

Figure 1: Top: A Kayak-paddle cooler [3]. At the lower part the sketch of more conservative design is represented.

In Fig. 1 straight sections are the same. Two of these sections joint by (semi) arcs. Straight sections made as a sequence of wigglers and accelerating structures, installed along straight line and having the bends at the end. Bends made with many short period divisions to prevent emittance dilution. Conservative scheme is more expensive, but, probably, is easy in tuning.

The cooler gives emittances obtained after considerations of radiation dynamics for a single electron in plane wiggler as [3]

$$
\begin{gathered}
\left(\gamma \mathcal{E}_{x}\right) \cong(1 / 2) \cdot \lambda_{C} \bar{\beta}_{x}\left(1+K_{x}^{2} / 2\right) K_{x} / \lambda \\
\left(\gamma \varepsilon_{y}\right) \cong(1 / 2) \cdot \lambda_{C} \bar{\beta}_{y} K_{x} / \lambda,
\end{gathered}
$$

where $\overline{\boldsymbol{\beta}}_{x, y}$-are averaged envelope functions in the wiggler. $K=e H_{\perp} \lambda_{w} / 2 \pi m c^{2}, H_{\perp}$-is the magnetic field in the wiggler, $\lambda_{w}$-is the wiggler period. The last formulas together with the cooling time

$$
c \tau_{\text {cool }} \cong(3 / 2) \cdot\left(\lambda_{w}^{2} / r_{0} K^{2} \gamma\right),
$$

defines the cooling dynamics under SR. Substitute for estimation $\bar{\beta}_{x, y} \approx 1 \mathrm{~m}, \lambda \cong 5 \mathrm{~cm}, K \cong 1$, one can obtain for quantum emittances the following

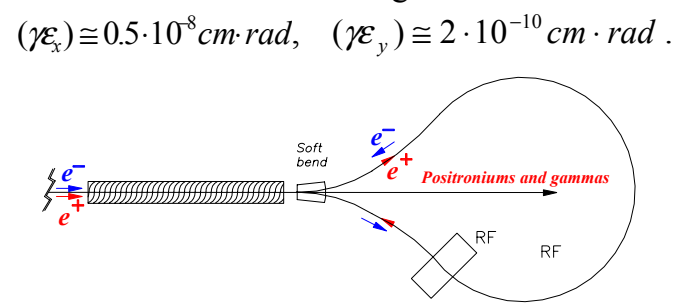

Figure 2: Bending area for co-directionally moving electrons and positrons. RF moved in arcs.

One can see that quantum limitations for the lowest emittance are important here. We also conclude, that coupling is allowable for the cooling business.

Motion in straight section filled with wigglers and $\mathrm{RF}$ cavities defined by specific lengthening is $\cong K^{2}$ times higher, than for free space,

$$
\kappa=\gamma \frac{\partial c t}{\partial \gamma}=\frac{\left(1+K^{2}\right) \cdot s}{4 \gamma^{2}}
$$

So the RF phase shift variation due to deviation of energy from equilibrium while passing the distance $d s$ is

$$
\delta \varphi=2 \pi \frac{\delta l}{\lambda}=\frac{2 \pi}{\lambda} \kappa \frac{\Delta \gamma}{\gamma} \cdot d s,
$$

where $\lambda$ is the wavelength of accelerating RF structure. As a result one can obtain the pendulum equation for energy deviation in terms of accelerating wavelength

$$
\frac{d^{2}(\Delta \gamma / \gamma)}{d^{2}(s / \lambda)}=-\frac{2 \pi e E \lambda \operatorname{Sin} \varphi_{0}}{m c^{2} \gamma} \cdot \frac{1+K^{2}}{\gamma^{2}} \cdot\left(\frac{\Delta \gamma}{\gamma}\right)
$$

By this procedure (wigglers and RF) longitudinal frequency can be kept high, so longitudinal motion can act on scene.

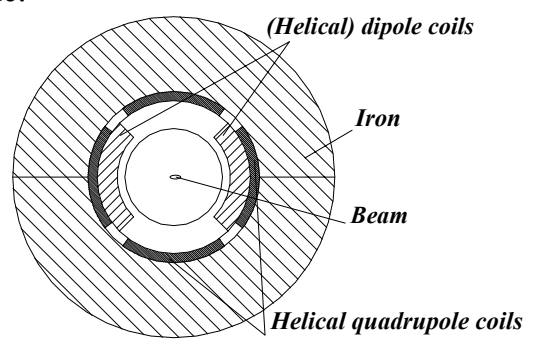

Figure 3: Elements of helical wiggler and helical quadrupole core cold mass. Beam is moving in vacuumed chamber. Windings done with SC wires. 
In addition to the helical dipole windings, the core contains the helical quadrupole windings too, Fig.3. Proposal to use helical quadrupole for particle confinement has a long history and was mostly in use in plasma business. Utilization of helical magnets for particle accelerators described in [8]. Usage of helical quadrupole and dipole is preferable due to simplified technology. Even in absence of artificial coupling there is a coupling by IBS like $\left(1+K_{x}^{2} / 2\right) \boldsymbol{\varepsilon}_{y} \boldsymbol{\beta}_{x}=\boldsymbol{\varepsilon}_{x} \boldsymbol{\beta}_{y}$, [4]. That is why full coupling allowed here.

\section{GENERATION OF POSITRONIUMS}

Cross section for para-positronium decay has dependence like $\sigma_{\uparrow \downarrow} \cong \pi r_{0}^{2} c / v_{\text {rel }}$, where $v_{\text {rel }}$ is relative velocity of particles [6]. Ortho-positronium decays in three gammas and has suppression factor as fine structure constant $\sigma_{\uparrow \uparrow} \cong \alpha \sigma_{\uparrow \downarrow}, \quad \alpha=e^{2} / \hbar c$. Transverse velocity in Lab frame is $v_{\perp} \equiv c K / \gamma$, where $K$ is undulatority factor defined earlier. So the cross section goes to

$$
\sigma_{\uparrow \downarrow} \cong \pi r_{0}^{2} \gamma / K \text {. }
$$

The luminosity for single bunch intrabeam collisions in a cooler could be estimated like [4]

$$
L \cong \frac{N^{2} \cdot c}{4 \pi l_{b} \lambda_{w} \sqrt{\left(\gamma_{y}\right) \beta_{y} / \gamma}} .
$$

For parameters considered above, (7) $\mathrm{L} \cong 10^{31} \mathrm{~cm}^{2} \mathrm{~s}^{-1}$. This defines the number of positroniums $\sim 10^{6} \mathrm{~s}^{-1}$.

Lifetime of the beam for gamma decay defined by lifetime of positroniums and by direct reaction of twophoton annihilation. The ratio between last ones defined by $\psi^{2}(0) / n_{b}$, where $\psi(r)=1 / \sqrt{\pi a} \exp (-r / a)$ is a psifunction of ground state of positronium, and $n_{b}$ stands for electron-positron density, $a=2 \lambda_{C} / \alpha$ is the Bohr's radius of positronium. Lifetimes of positroniums $\tau_{\uparrow \downarrow} \cong 2 \hbar / m c^{2} / \alpha^{5} \approx 1.2310^{-10} \mathrm{~s}$ (para, two gammas), $\tau_{\uparrow \uparrow} \cong 1.4 \cdot 10^{-7} \mathrm{~s}$, (ortho, three gammas).

If positronium is not decayed and coming to splitting magnet it can simply continue straight motion out of the ring or be split for electron and positron. The field value in soft bend (Fig.2) must satisfy condition $H \gamma \leq e / a^{2}$. This gives $H \leq 4.4 \cdot 10^{6} / \gamma[\mathrm{G}]$. Otherwise magnetic field of magnet will pull apart electron and positron.

\section{EXTREME RING}

Now we are ready to introduce some extreme configuration dedicated exclusively for the purposes of positronium generation Fig. 4. This is basically the same straight sections as in Fig.1 arranged, however for electrons and positrons have individual channels.

Operation of this ring is clear from described above, however. In helical wiggler (undulator) positrons and electrons are rotating in the same direction, what gives same preference with solenoid.
For injection usual hardware can be used. Positrons and electrons obtained in external accelerators, not shown here for simplicity and trivial nature (the same for Fig. 1).

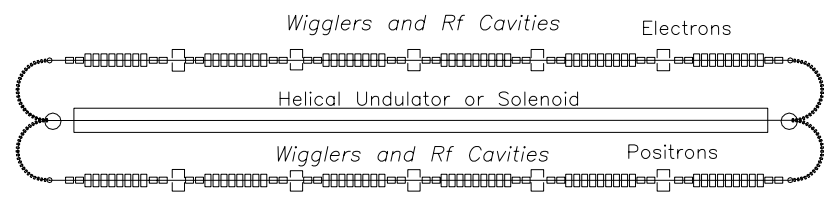

Figure 4: Extreme ring for demonstration of Fermidegeneration and positronium generation.

This ring gives one ray of gammas and positroniums. Interest for positronium generation is shown in [9] also.

\section{CONCLUSION}

In this publication we introduced a plan for dedicated ring construction for investigation of Fermi degeneration in moving beams. This is a part of big scheme for obtaining the ultra-cold beams allowing drastic decrease of number of particles required for collision and/or reduction of the acceleration wavelength.

The work supported by NSF.

\section{REFERENCES}

[1] A. Mikhailichenko, "On the physical limitation to the lowest emittance (toward colliding electron- positron crystalline beams)", $7^{\text {th }}$ Advanced Accelerator Concepts Workshop, 12-18 October 1996, Lake Tahoe, CA, AIP Conference Proceedings 398, p.294: CLNS 96/1437, Cornell, 1996.

[2] A. A. Mikhailichenko, "To the quantum limitations in beam physics", CLNS99/1608,March 23, 1999; a paper at PAC99, Proc., vol. 4, pp. 2814-2816.

[3] A.A. Mikhailichenko, "Damping Ring for VLEPP linear collider", III international Workshop on linear Colliders LC91, Protvino, September 17-27, 1991. Proceedings, Edited by V.E. Balakin, S. Lepshokov, N.A. Solyak, Serpukhov, (IFVE). 1991. Serpukhov, USSR: BINP (1991).

[4] A. Mikhailichenko, "Injector for a Laser Linear Collider", CLNS 98/1568, a Talk on $8^{\text {th }}$ Advanced Accelerator Concepts Workshop, Baltimore, Maryland, July 5-11, 1998, pp.891-900..

[5] L.D. Landau, E.M. Lifshitz, "Statistical Physics", Pergamon Press, 1985.

[6] V.B. Berestetskii, E.M. Lifshits, L.P. Pitaevskii, "Quantum Electrodynamics", Pergamon Press, Second Edition, 1982.

[7] A. Piwinsky, "IBS", Proc. 9th Intern. Conf. on High Energy Acc., Stanford, CA, 2-7 May, 1974, SLAC, 1974, p.405.

[8] A.S. Artamonov, Ya.S. Derbenev, N.I. Inozemtsev, "Helical achromatic focusing of a charged particle beam", Zh.Tekh. Fiz. 60, 156-159 (1990). Transl.: Sov. Tech. Phys. 35(4) 1990.

[9] I.N.Meshkov, A.N. Skrinsky, NIM A391(1997) 205209. 